\title{
Pulse Compression Method for Radar Signal Processing
}

\author{
Thin Thin Mar \\ Department of Electronic Engineering \\ Mandalay Technological University \\ Mandalay, Myanmar
}

\author{
$\mathrm{Su} \mathrm{Su}$ Yi Mon \\ Department of Electronic Engineering \\ Mandalay Technological University \\ Mandalay, Myanmar
}

\begin{abstract}
One fundamental issue in designing a good radar system is it's capability to resolve two small targets that are located at long range with very small separation between them. Pulse compression techniques are used in radar systems to avail the benefits of large range detection capability of long duration pulse and high range resolution capability of short duration pulse. In these techniques a long duration pulse is used which is frequency modulated before transmission and the received signal is passed through a match filter to accumulate the energy into a short pulse. A matched filter is used for pulse compression to achieve high signal-to-noise ratio (SNR). Two important factors to be considered for radar waveform design are range resolution and maximum range detection. Range resolution is the ability of the radar to separate closely spaced targets and it is related to the pulse width of the waveform. The narrower the pulse width the better is the range resolution. But, if the pulse width is decreased, the amount of energy in the pulse is decreased and hence maximum range detection gets reduced. To overcome this problem pulse compression techniques are used in the radar systems. In this paper, the pulse compression technique is described to resolve two small targets that are located at long range with very small separation between them.
\end{abstract}

Keywords: Pulse compression, Radar, LFM, Matched filter, Range Resolution

\section{INTRODUCTION}

The rapid advances in digital technology made many theoretical capabilities practical with digital signal processing and digital data processing. Radar signal processing is defined as the manipulation of the received signal, represented in digital format, to extract the desired information whilst rejecting unwanted signals. Pulse compression allowed the use of long waveforms to obtain high energy simultaneously achieves the resolution of a short pulse by internal modulation of the long pulse. The resolution is the ability of radar to distinguish targets that are closely spaced together in either range or bearing. The internal modulation may be binary phase coding, polyphase coding, frequency modulation, and frequency stepping. There are many advantages of using pulse compression techniques in the radar field. They include reduction of peak power, relevant reduction of high voltages in radar transmitter, protection against detection by radar detectors, significant improvement of range resolution, relevant reduction in clutter troubles and protection against jamming coming from spread spectrum action [1].

Pulse compression employs a modulated "long" pulse to achieve the range resolution of a "short" pulse without the need for high peak transmit power. Short pulses are better for range resolution, but contradict with energy, long range detection, carrier frequency and SNR. Long pulses are better for signal reception, but contradict with range resolution and minimum range. At the transmitter, the signal has relatively small amplitude for ease to generate and is large in time to ensure enough energy in the signal. At the receiver, the signal has very high amplitude to be detected and is small in time.

In pulse compression technique, the transmitted signal is frequency or phase modulated and the received signal is processed using a specific filter called "matched filter". A matched filter is a linear network that maximizes the output peak-signal to noise ratio of a radar receiver which in turn maximizes the detect ability of a target. There are several methods of pulse compression that have been used in the past. The most popular of them is linear frequency modulation (LFM) which was invented by R.H. Dickie in 1945 [1]. The other pulse compression techniques are Binary phase codes, Polyphase codes, Barker codes, Costas codes, Nonlinear Frequency Modulation etc. In this paper, we developed MATLAB code to study the LFM pulse compression technique with chirp diversity.

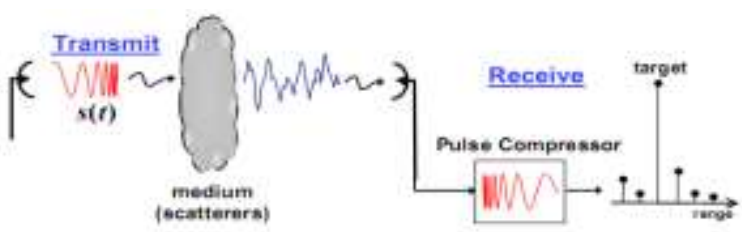

Figure 1. Pulse Compression

\section{PULSE COMPRESSION ALGORITHM}

The maximum detection range depends upon the strength of the received echo. To get high strength reflected echo the transmitted pulse should have more energy for long distance transmission since it gets attenuated during the course of transmission. The energy content in the pulse is proportional to the duration as well as the peak power of the pulse. The product of peak power and duration of the pulse gives an estimate of the energy of the signal. A low peak power pulse with long duration provides the same energy as achieved in case of high peak power and short duration pulse. Shorter duration pulses achieve better range resolution. The range resolution $r_{\text {res }}$ is expressed [2] as

$\mathrm{r}_{\mathrm{res}}=\frac{\mathrm{c}}{2 \mathrm{~B}}$

where $\mathrm{B}$ is the bandwidth of the pulse.

For unmodulated pulse, the time duration is inversely proportional to the bandwidth. If the bandwidth is high, then the duration of the pulse is short and hence this offers a superior range resolution. Practically, the pulse duration cannot be reduced indefinitely. According to Fourier theory a signal with bandwidth B cannot have duration shorter than 
1/B i.e. its time-bandwidth (TB) product cannot be less than unity. A very short pulse requires high peak power to get adequate energy for large distance transmission. However, to handle high peak power the radar equipment become heavier, bigger and hence cost of this system increases. Therefore peak power of the pulse is always limited by the transmitter. A pulse having low peak power and longer duration is required at the transmitter for long range detection. At the output of the receiver, the pulse should have short width and high peak power to get better range resolution. Figure 2 illustrates two pulses having same energy with different pulse width and peak power. To get the advantages of larger range detection ability of long pulse and better range resolution ability of short pulse, pulse compression techniques are used in radar systems [2].

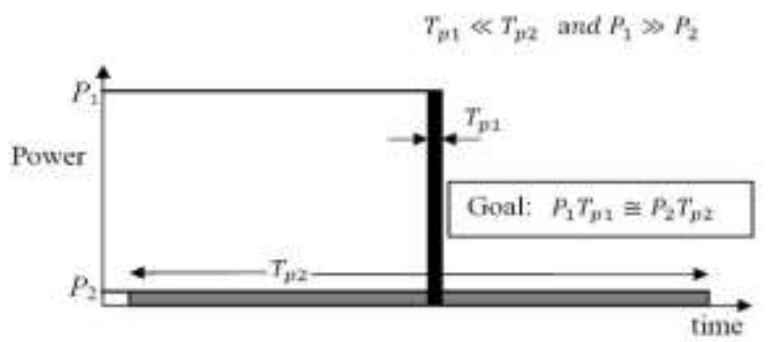

Figure 2. Transmitter and receiver ultimate signals

The range resolution depends on the bandwidth of a pulse but not necessarily on the duration of the pulse [3]. Some modulation techniques such as frequency and phase modulation are used to increase the bandwidth of a long duration pulse to get high range resolution having limited peak power. In pulse compression technique a pulse having long duration and low peak power is modulated either in frequency or phase before transmission and the received signal is passed through a filter to accumulate the energy in a short pulse. The pulse compression ratio (PCR) is defined as

$$
\mathrm{PCR}=\frac{\text { width of the pulse before compression }}{\text { width of the pulse after compression }}
$$

\subsection{LFM Algorithm}

Linear frequency modulated (LFM) signals are used in most of the radar systems to achieve wide operating bandwidth. In this case the frequency increases (up chirp) or decreases (down chirp) linearly across the pulse. The instantaneous phase of the chirp signal is expressed as

$$
\varphi(t)=2 \pi\left(\mathrm{f}_{0} \mathrm{t}+\frac{1}{2} \mathrm{kt}^{2}\right)
$$

where $\mathrm{f}_{0}$ is the carrier frequency and $\mathrm{k}$ is the frequency sweep rate related to pulse duration $T_{p}$ and bandwidth $B$ as

$$
\mathrm{k}=\frac{\mathrm{B}}{\mathrm{T}_{\mathrm{p}}}
$$

The instantaneous frequency is given by

$$
\mathrm{f}(\mathrm{t})=\frac{\mathrm{d}}{\mathrm{dt}}\left(\mathrm{f}_{0} \mathrm{t}+\frac{1}{2} \mathrm{kt}^{2}\right)=\mathrm{f}_{0}+\mathrm{kt}
$$

Equation (5) states that the instantaneous frequency is a linear function of time, and hence is called as linear frequency modulation. Figure 2 (a) shows the modulated sinusoidal signal that is transmitted by the pulse-compression radar. The pulse is characterized by its pulse width, which in the case of a pulse-compression radar is called the uncompressed pulse width, T. This pulse width is one of the critical characteristics of the pulse-compression radar.
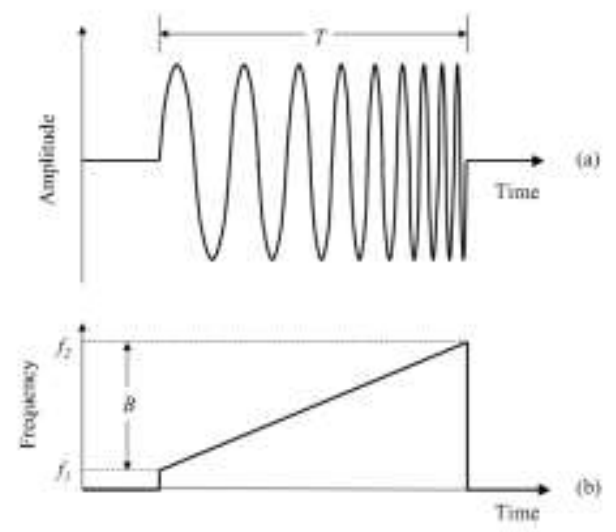

Figure 2. The amplitude of (a) a frequency-modulated pulse over time and (b) the frequency of the same pulse over time.

Figure 2(a) illustrates the instantaneous frequency of LFM waveform that sweeps from $f_{0}$ to $f_{1}$. The characteristic of interest in Figure 2(b) is the bandwidth of the modulation within the pulse, B. The bandwidth is simply the difference between the highest and lowest frequencies within the uncompressed pulse.

To recognize the presence of the uncompressed pulse, the pulsecompression filter performs a correlation between the received pulse and the transmitted pulse. The pulse-compression filter (matched filter) is simply looking for a strong correlation between what was transmitted and what was received.

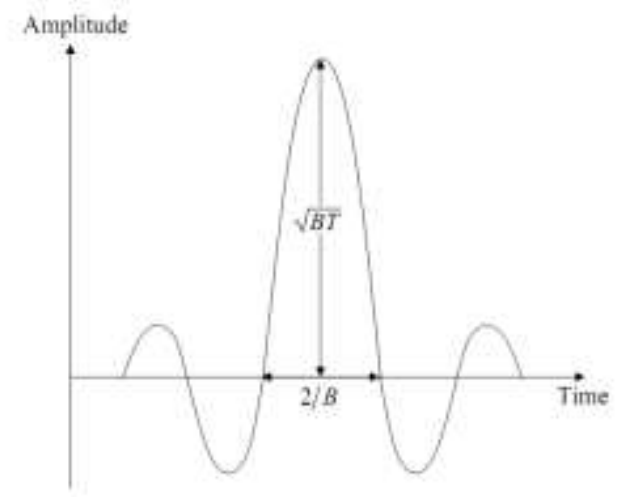

Figure 3. Typical output from a pulse-compression filter

The output of the pulse-compression filter forms the input into the detector section of the pulse-compression radar. It is therefore desirable to have a very narrow and tall pulse. The main points in Figure 3 are that the input to the filter is a relatively wide and low- power pulse. The output pulse, however, is very narrow and strong; two very desirable characteristics from a pulse radar.

The output of the pulse-compression filter shown in Figure 3 represents the amplitude of a signal rather than its power. To be consistent with the radar range equation, the output of the pulse-compression filter is converted into power that is taken as the square of the amplitude. When the signal in Figure 3 is converted into power, the peak value of the pulse becomes the product of the modulation bandwidth, B and the uncompressed pulse width, T. This is known as the pulse-compression ratio of the pulse-compression radar.

Pulse compression ratio $=\mathrm{BxT}$

where $\mathrm{B}$ is the bandwidth of the modulation within each pulse in hertz and $\mathrm{T}$ is the uncompressed pulse width in seconds. 
The width of the compressed pulse is simply the inverse of the modulation bandwidth, $\mathrm{B}$.

Compressed pulse width $=1 / \mathrm{B}$

where $\mathrm{B}$ is the bandwidth of the modulation within each pulse in hertz.

\subsection{Matched Filter}

In radar applications the reflected signal is used to determine the existence of the target. The reflected signal is corrupted by additive white Gaussian noise (AWGN). The probability of detection is related to signal-to-noise ratio (SNR) rather than exact shape of the signal received. Hence it is required to maximize the SNR rather than preserving the shape of the signal. A filter which maximizes the output SNR is called matched filter [5].

Pulse compression radar is the practical implementation of a matched-filter system. The coded signal can be described either by the frequency response $\mathrm{H}(\omega)$ or as an impulse response $h(t)$ of the coding filter. The received echo is fed into a matched filter whose frequency response is the complex conjugate $H^{*}(\omega)$ of the coding filter. The output of the matched filter, $y(t)$ is the compressed pulse which is just the inverse Fourier transform of the product of the signal spectrum and the matched filter Response.

$y(t)=\frac{1}{2 \pi} \int_{-\infty}^{\infty}|H(\omega)|^{2} \exp (j \omega x) d \omega$

A filter is also matched if the signal is the complex conjugate of the time inverse of the filter's impulse-response. This is often achieved by applying the time inverse of the received signal to the pulse-compression filter. The output of this matched filter is given by the convolution of the signal $h(t)$ with the conjugate impulse response $h *(t)$ of the matched filter

$\mathrm{y}(\mathrm{t})=\int_{-\infty}^{\infty} \mathrm{h}(\tau) \mathrm{h} *(\mathrm{t}-\tau) \mathrm{d} \tau$

\section{SIMULATION RESULTS}

Firstly, for the testing purpose of simulation certain LFM pulse waveform is generated with the following characteristics:

Sample rate $=1 \mathrm{e} 6$

Duration of a single pulse $=100 \mathrm{e}-6$

Pulse repetition frequency $=5 \mathrm{e} 3$

Sweep bandwidth 1e5

Sweep direction=up

The parameters are defined based on the specifications of some radar simulation project. Figure 4 shows an uncompressed chirp pulse representing the transmitted pulse. Noise and attenuation are a real problem when operating radar systems, so both random noise and signal attenuation are incorporated, and is shown in Figure 5. The output of the corresponding pulse-compression filter is shown in Figure 6 when that pulse is processed.

The two graphs in Figure 4 and Figure 6 are drawn on the same time scale (horizontal axis) to show how the process has compressed into a very narrow pulse. It is not possible to draw the amplitude axes (vertical axis) on the same scale due to the magnitude difference between Figure 4 and 6 . The amplitude of the pulse in Figure 6 is approximately 100 times larger than Figure 4. Although the noise effects on the input pulse, the output is still impressive from a peak amplitude and pulsewidth perspective and the target is clearly visible.

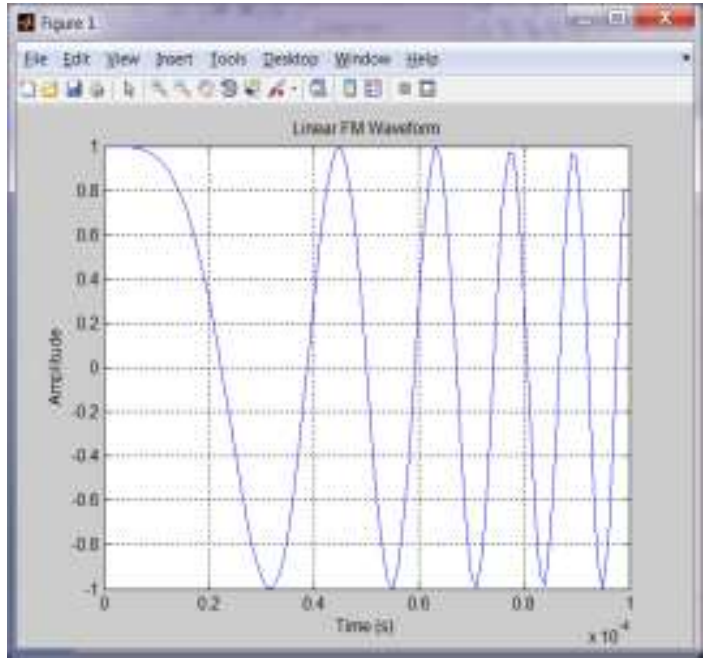

Figure 4. LFM Uncompress Pulse

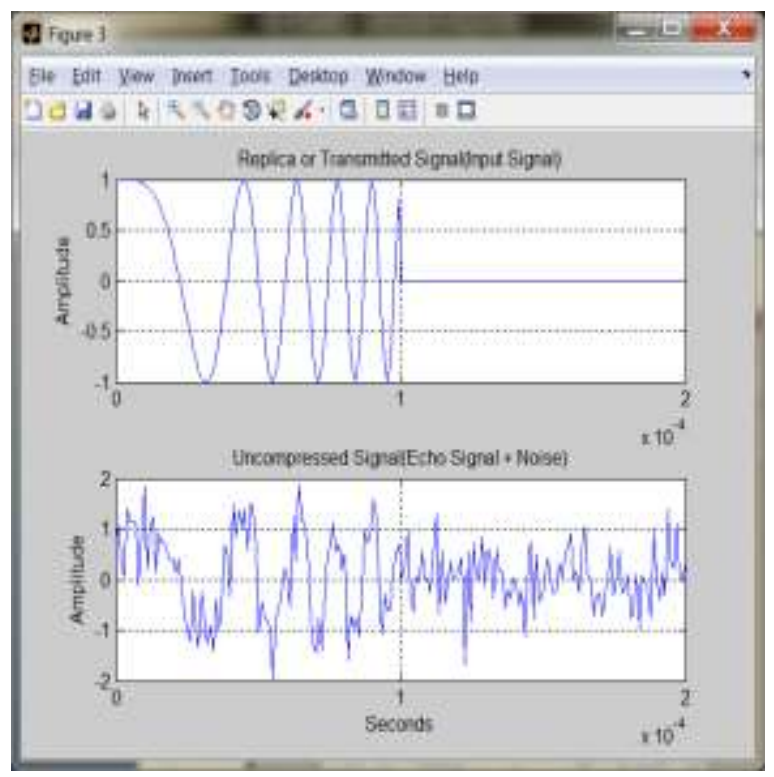

Figure 5. LFM Uncompress Pulse and Noise Signal

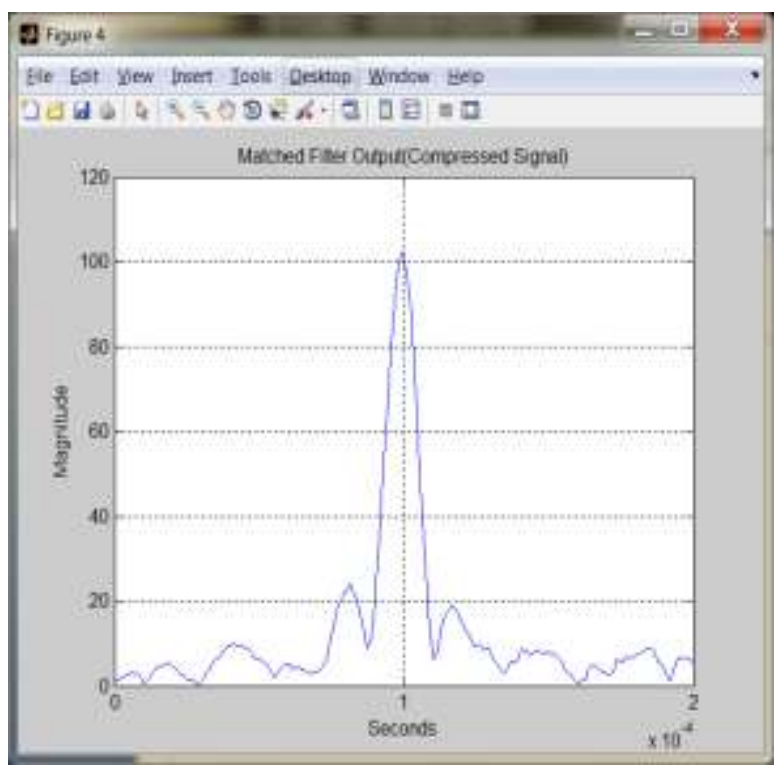

Figure 6. Compressed Pulse 
Secondly, the linear FM waveform is configured with the following characteristics. The desired range resolution determines the waveform bandwidth. For a linear FM waveform, the bandwidth is equal to its sweep bandwidth. However, the pulse width is no longer restricted to the reciprocal of the pulse bandwidth, so a much longer pulse width can be used. We use a pulse width that is 20 times longer and set the sample rate to be twice the pulse bandwidth.

The input parameters for the simulation are

Number of targets within the radar's maximum rage $=3$

Radar's maximum range $=6000 \mathrm{~m}$

Range Resolution $=50$;

Chirp bandwidth $=\mathrm{c} /(2 *$ range_res $)=30 \mathrm{MHz}$

Uncompressed Pulse Width (seconds) = 20/BW;

Sampling Frequency $=2 * \mathrm{BW}$;

Targets' relative range $(\mathrm{m})=2000,3500,3600$

Figure 7 illustrates the created LFM waveform. Figure 8 is simulation result of uncompressed echo in time domain (seconds). Scatters are not resolved. Figure 9 illustrates the compressed echo signal in target position (meters). Two targets that are located at long range with very small separation (100 meters) between them are resolved.

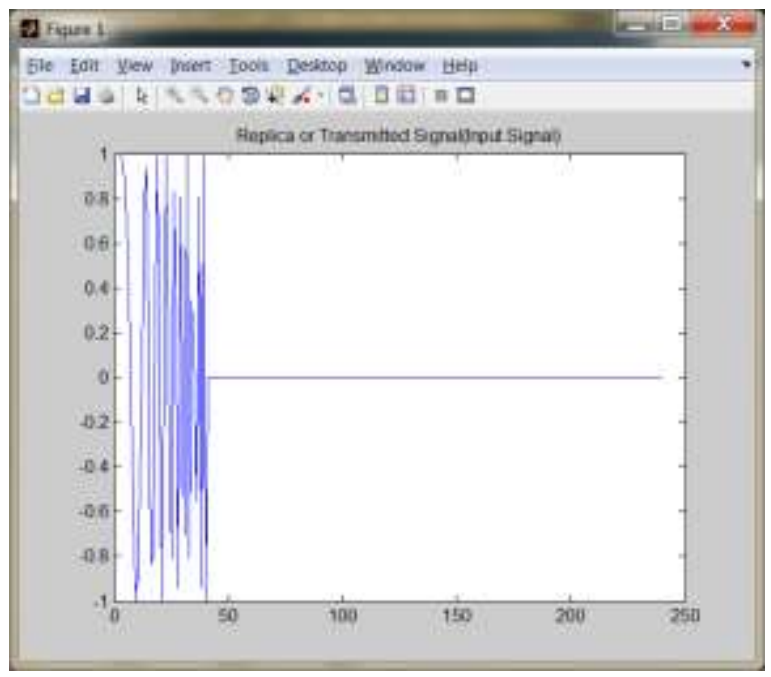

Figure 7. Transmitted LFM pulse

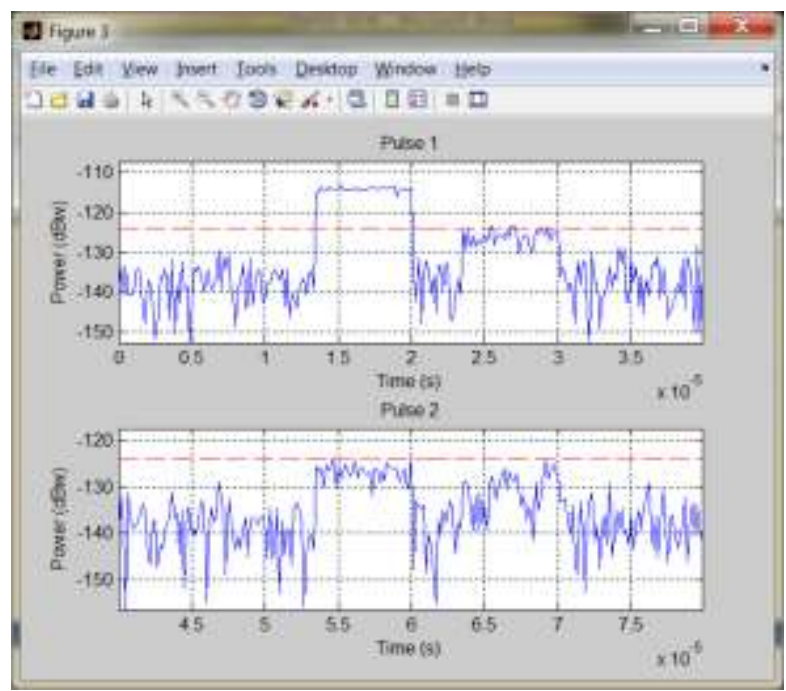

Figure 8. Received signal (Uncompressed echo)

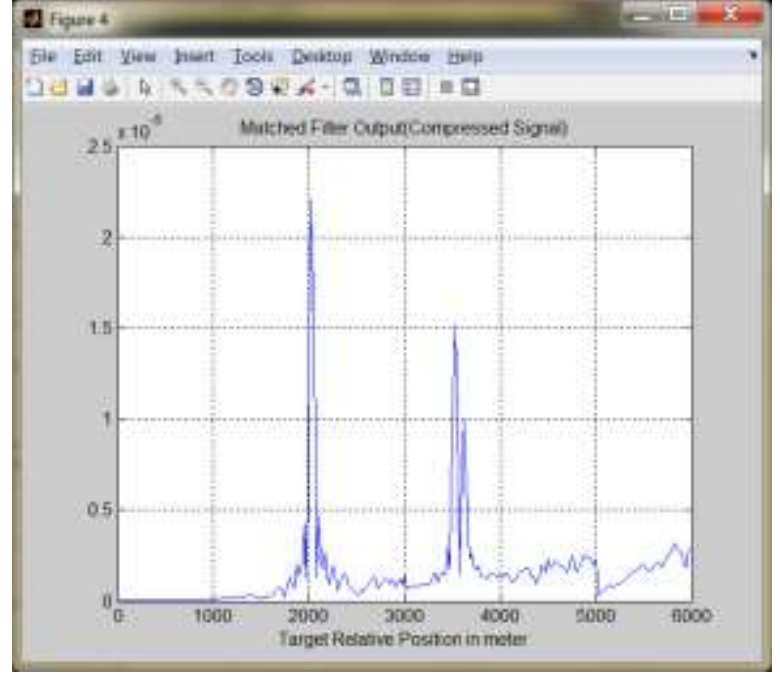

Figure 9. Compressed echo

And then, the program is simulated by setting the two targets in the range 3500 and 3560 meters. When two targets are separated by 60 meters, it can resolve completely as shown in Figure 10

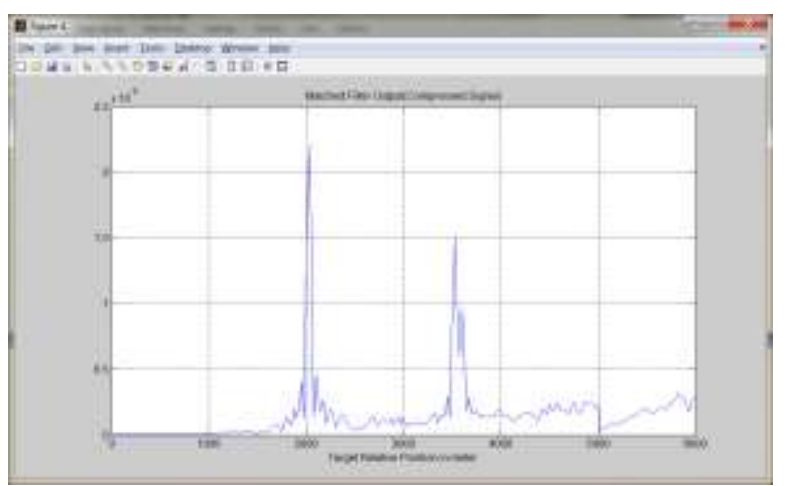

Figure 10. Compressed echo

Figure 11 is similar to Figure 10 except in this case the second and third targets are less than range resolution apart (They are at 3500 and 3540 meters from radar)

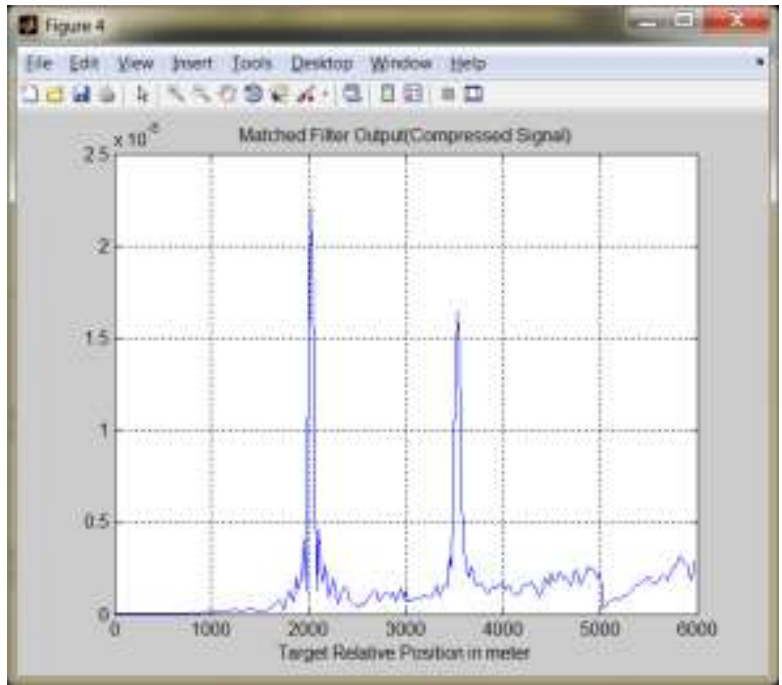

Figure 11. Compressed echo of two unresolved targets 


\section{CONCLUSIONS}

The concept of pulse-compression was presented in this paper using the linear FM (or chirp) pulse modulation. In this method, received signal is compared to matched filter impulse response for correlation. A compression of the effective pulse width of received pulses leading to range advantages and range resolution improvements. The effectiveness of pulsecompression is determined by the width of the uncompressed pulse and the bandwidth of the modulation within the uncompressed pulse. The most impressive results come from broad uncompressed pulse widths containing a modulated pulse with a large bandwidth. Range resolution is the ability to separate multiple targets at different ranges. To be resolved in range, the basic criterion is that targets must be separated at least in the range equivalent of the width of the processed echo pulse. In this paper, it is shown that the distance between two targets less than range resolution and the targets are not resolved.

\section{REFERENCES}

[1] Skolnik, M.I., Introduction to Radar Systems, Sydney: McGraw-Hill, 1988, p. 434.

[2] D.K. Barton, Pulse Compression. Artech House, 1975.

[3] A.W. Rihaczek, Principle of high resolution radar. McGraw Hill, New York, 1969.

[4] Stimson, G.W., Introduction to Airborne Radar, New Jersey: SciTech Publishing Inc., 1998, p. 393.

[5] Jane's Radar and Electronic Warfare Systems 1999-00, AN/APS- 134(V)/-134 (Plus) Maritime Surveillance Radars, Jane's Information Group, 1999.

[6] B. L. Lewis, F. F. Kretschmer Jr., "Linear Frequency Modulation Derived Polyphase Pulse Compression Codes", IEEE Trans. on Aerospace and Electronic Systems, vol. AES-18, no. 5, pp. 637-641, Sep. 1982.

[7] F.E. Nathanson, J. P. Reilly and M. N. Cohen, "Radar Design Principles Signal Processing and the Environment", $2^{\text {nd }}$ ed. New York: McGraw-Hill, 1999, chapt. $1 \& 8$. 